\title{
Luteal phase pregnancy after mirena coil insertion for intrauterine septum resection
}

\author{
Natasha Louise Citeroni ${ }^{1}$, Rifat Syed ${ }^{2}$
}

'GKT School of Medical Education, King's College London, Great Maze Pond, London, United Kingdom

${ }^{2}$ Department of Obstetrics \& Gynaecology, Darent Valley Hospital, United Kingdom

\section{Corresponding Author}

Natasha Louise Citeroni. GKT School of Medical Education, King's College London, Great Maze Pond, London, SE1 1UL, UK, e-mail: natasha.citeroni@kcl.ac.uk

\section{Abstract}

This report is of an unusual case of an intrauterine singleton pregnancy with a Mirena Coil (IUS) in situ following an elective hysteroscopic intrauterine septum resection for treatment of primary subfertility in a 27-year-old female. The patient elected to continue with the pregnancy and underwent additional monitoring via ultrasound. A caesarean section was undertaken at 39-weeks gestation and a healthy male infant was born. The Mirena coil was recovered intraoperative and was visualised in the placental membranes. Pregnancy occurring with Mirena coil insertion as an adjunct to hysteroscopic septum resection in our case is an unusual clinical scenario with no management recommendations and limited recorded outcomes. Therefore, this case demonstrates potential management of such a case and learning points for intrauterine septum excision in the context of primary subfertility.

Key words: Pregnancy, hysteroscopy, uterine septum resection, Mirena, IUS, Subfertility

\section{Background}

Hysteroscopic resection is the preferred secondary care treatment for the presence of a uterine septum which is a recognised cause of recurrent miscarriages. It involves metroplasty of an anomalous septum with the aim of re-forming the normal uterine anatomy and facilitating successful implantation. The Mirena coil is used as a hormonal adjunct to facilitate improvement in the histology of the endometrium to favour implantation. In this case, an early luteal pregnancy was present at the time of insertion creating both ethical and management challenges regarding whether or not removal of the Mirena should be attempted.

\section{Case Presentation}

A 26-year-old well Caucasian female patient presented to the subfertility clinic at a District General hospital after GP referral from primary subfertility for 3 years, with no significant co-morbidities. Basic 
investigations such as blood tests had shown no abnormality with normal hormone profile, the following levels present: FSH 6.9, LH of 3.4, Prolactin of 672 and Serum Testosterone of 0.7. The patient was having regular periods. Semen Analysis of her partner was also normal.

The patient undertook further investigation for her subfertility and was consented to outpatient hysterosalpingo-contrast-sonography (HyCoSy) with saline sonography which demonstrated an anteverted normal sized uterus with patent fallopian tubes. However, a uterine septum of approximately 1.5 $\mathrm{cm}$ was present and was confirmed with a 3D scan.

The patient consented for elective hysteroscopic septum resection and hysteroscopy confirmed the presence of a uterine septum which was successfully resected using a resectoscope. To prevent adhesion formation, a Mirena coil was inserted which was due to be removed 8-weeks post-procedure. Before the Mirena was inserted a pregnancy test was performed which was negative and the patient's last menstrual period (LMP) was 3 weeks prior to insertion. The patient was called for follow up after approximately 3 weeks and was noted to have missed her period. There was no history of any sexual intercourse post hysteroscopy.

\section{Investigations}

The patient took a home pregnancy test following advice from secondary care and then presented to the Early Pregnancy Unit for a 6-week transvaginal scan. This confirmed an intrauterine pregnancy estimated to be 6 weeks gestation with a Crown-RumpLength (CRL) of $0.34 \mathrm{~cm}$. At this time, the Mirena was located close to the gestational sac (Figure 1). This was significant to rule out the pregnancy as ectopic, common association with the Mirena coil ${ }^{1}$. It was noted at this time the Mirena threads were unable to be located.

The patient was transferred to Obstetric care for further investigation and management. Obstetrically, there were only minimal complications such as a minor self resolving per vaginal bleed in the first trimester as well as a noted low PAPP-A value. The Mirena was able to be visualised in the subchorionic position at the 12-week scan (Figure 2). On scanning throughout, all scans showed appropriate foetal development with no structural abnormalities. The patient then had a subsequent follow-up scan in which the Mirena was unable to be located, which lead to monthly ultrasound scans for additional monitoring. In the following scans the Mirena was believed to be in the lower third of the uterus. (Figures 3 and 4).

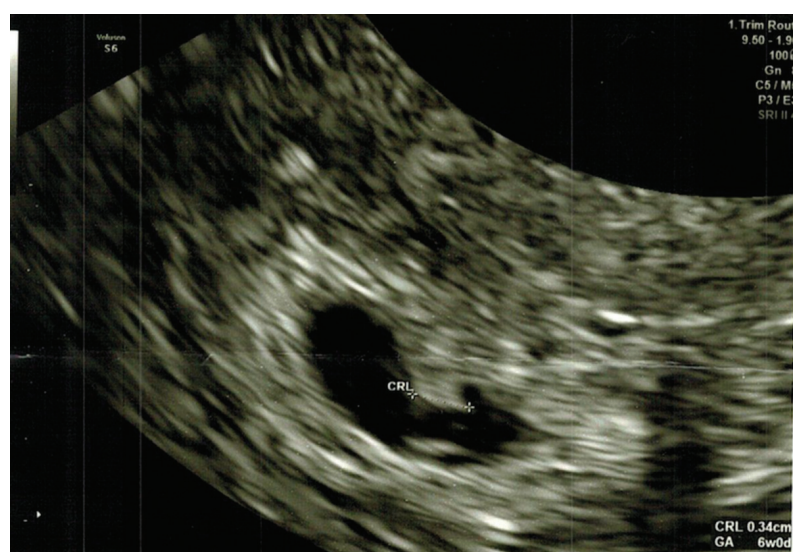

Figure 1. 6-week scan.

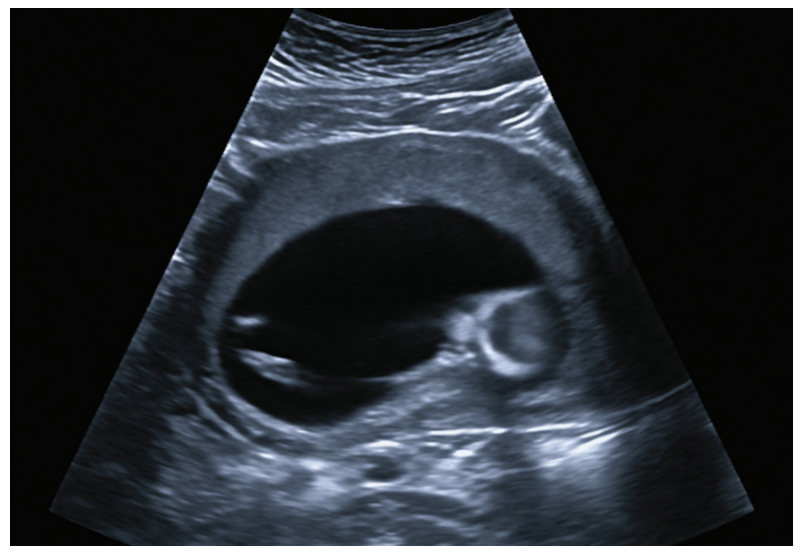

Figure 2. 12-week scan. 


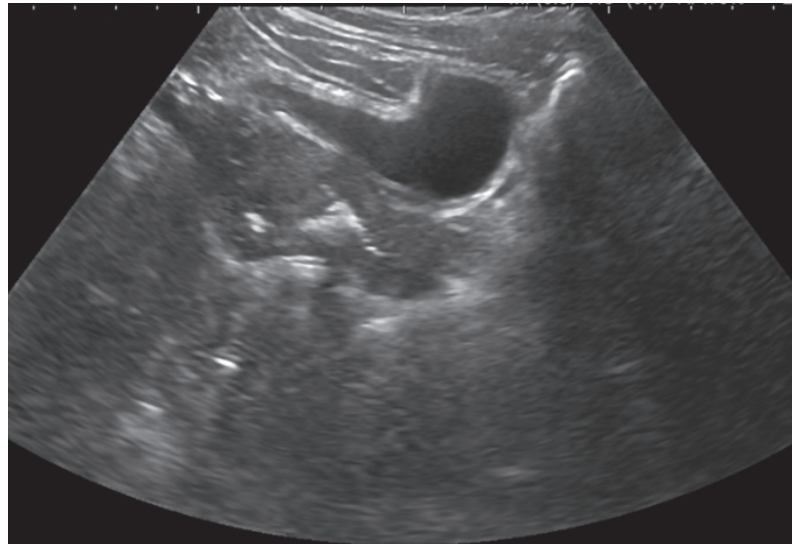

Figure 3. 28-week scan.

\section{Treatment}

Regarding birth planning and management of her pregnancy, mode of delivery was discussed. The patient decided to undergo an elective caesarean section due to her high risk status as the pregnancy had implanted following the resection. The foetus was delivered by cephalic presentation via caesarean section at 39-weeks gestation and the Mirena coil was recovered from the membranes in the placenta. The neonate was reviewed by the neonatal team at the time of delivery and was assessed to be normal. Estimated blood loss was within normal ranges and the uterine cavity was found regular and smooth. The patient was given post operative analgesia and thromboprophylaxis as per the general management of a post caesarean section

\section{Follow up and Outcome}

The patient was debriefed regarding the surgical outcomes and recovery of the Mirena coil after the caesarean section. She was then discharged home the next day to midwifery lead care in the community, as per the standard management of a primiparous woman.

\section{Discussion}

Hysteroscopic metroplasty, including transcervical

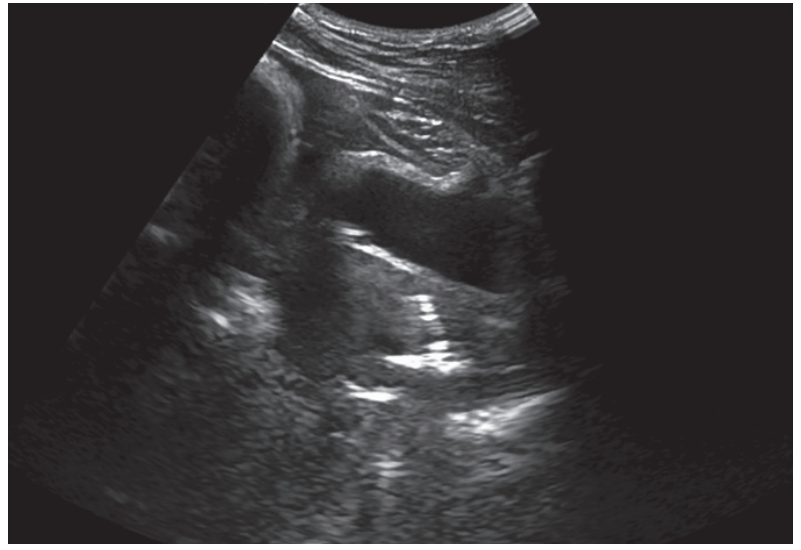

Figure 4. 30-week scan.

resection or division is the treatment of choice for uterine anomalies including uterine sepal defects, which is the uterine anomaly with the highest prevalence, as suggested by a number of studies ${ }^{2,3}$. The procedure is superior to transabdominal resections due to its less risk of complications, adhesion formation, hospital stay length, as well as a significantly reduced blood loss and is therefore a cost effect procedure with low morbidity for otherwise well patients ${ }^{4}$.

It is well documented that hysteroscopic metroplasty appears to be beneficial in reducing miscarriage rates in women with a septate uterus, however, whether the procedure has the same level of benefit for women presenting with primary subfertility is less clear ${ }^{5}$. Nevertheless, the procedure is commonly performed, particularly in the instance of unexplained subfertility when no other abnormalities can be found in the hope of improving the odds of a viable pregnancy. With many cases reporting success in conception rates after septal dissection ${ }^{6,7}$. The believed pathophysiology for this is largely based on the poor response to oestrogen which has been demonstrated in septal tissue ${ }^{8}$. Thus it has been postulated that implantation may be hindered by the avascular nature of the septum as well as be- 
ing responsible for poor placental growth leading to increased miscarriage rates among this cohort ${ }^{9}$.

As well as septal resection itself, there are a number of hormonal and barrier adjuncts to the procedure that can be performed under hysteroscopy in the aim of preventing adhesion formation following resection. Adhesion formation can reduce fertility potential and has been associated with increased miscarriage rates and pregnancy morbidity, as seen in Asherman's syndrome ${ }^{10}$. Which is a potential and significant complication of the procedure given that many patients present for treatment of subfertility or recurrent miscarriage.

The effectiveness and possible complications of hysteroscopic metroplasty have never been tested in randomised control trials. Furthermore, there is varying debate over which specific adjunctive therapy is most effective in preventing adhesion formation, or if any of the current treatment options offer any real protection against adhesion development $t^{11,12,13}$.

Despite the lack of conclusiveness and varying debate surrounding the use of adjuncts, many physicians still chose to use these based on their prior use in laparoscopy in the hope that they may offer some benefit even if significant improvements have not been proven. In a recent Cochrane review of 560 women across 8 studies fewer intrauterine adhesions were demonstrated at second-look hysteroscopy when using an adjunct compared with no treatment or placebo (OR $0.35,95 \%$ CI 0.21 to $0.60)$. However, the quality of evidence here was considered to be low $^{14}$. In this case, as the patient achieved pregnancy, the assessment of the Mirena for the intended benefit of reducing adhesions is not possible to fully evaluate. Although, during the caesarean section in our case, the uterus was regarded as smooth with no palpable adhesions or evidence of pathology on surgical examination. The National Institute for Health and Care Excellence (NICE) have recently produced guidance on hysteroscopic metroplasty of uterine septum for recurrent miscarriage and for primary subfertility, however, this also considers the relative lack of evidence on the subject ${ }^{15}$.

True Mirena coil failure is rare, with an estimated 1- year Pearl rate of 0.1 in the literature, with $53 \%$ of those pregnancies being ectopic in a large study of 17,360 users ${ }^{1}$. However, this conception occurred before insertion and as such there is no available data on the rates of successful implantation once a Mirena has been inserted in such a scenario. Regarding the risks of an intrauterine pregnancy with a Mirena in-situ, when the pregnancy was discovered the patient was presented with the available options and counselled appropriately on the potential risk of miscarriage if the Mirena was removed, as well as risks with continuing the pregnancy with the Mirena in-situ such as: preterm labour and infection (including septic miscarriage). In addition to the potential risk of congenital malformation or virilisation of the foetus, which is generally thought of to be extremely low despite theoretical concerns ${ }^{16}$.

\section{Conclusion}

This report is of an unusual case of an early luteal pregnancy occurring with a Mirena coil in situ following hysteroscopic septum resection in a 27-year-old female. A literature search undertaken by our team suggested that no such case has previously been reported raising a gap in the literature surrounding this potential scenario and its management, for which further research is required. We also conclude that large scale Randomised Controlled Trials are also needed to fully assess the benefits of both uterine septum resection and the role for adjunctive treatments in pregnancy outcomes in primary subfertility patients.

Furthermore, it is positive in this case that the Mirena coil insertion did not appear to have any det- 
rimental or structural effects on the foetus involved. Although more research is needed as currently, due to the rarity of this scenario, the sample sizes of previously reported cases are low ${ }^{2}$.

\section{Learning Points}

- In this case, the negative pregnancy test on Mirena insertion was taken as read. Resultantly, patients will now be advised to not have any unprotected intercourse from their last menstrual period until after their resection once the Mirena has been inserted. Or, to use another method of contraception such as the contraceptive pill.

- In addition, the aim will now be to perform the procedure during the early follicular phase of the woman's menstrual cycle in order to further safeguard against the risk of pregnancy.

- Patients will now also be counselled about the risk of pregnancy in the presence of the Mirena coil as a potential risk of non-abstinence and/or contraceptive failure in this period.

- As a final learning point following review of the literature for this case report we recognise the need to consider a patient's suitability for the Mirena coil as part of the treatment for a uterine septum resection and the potential to use other hormonal adjuncts such as oestrogen as an alternative to promote endometrial growth, without the risk of a subsequent complicated pregnancy.

\section{Acknowledgements}

The authors would like to thank the patient whom gave written consent for the use of their images and medical records for the publication of this case report.

The authors would also like the thank the on-site Clinical Library for the following evidence search: Uterine septum resection and Mirena and pregnancy. Genny Franklin: (4 ${ }^{\text {th }}$ October, 2019), Dartford and Gravesham NHS Trust, UK.

\section{Disclosure}

The authors report no conflicts of interest in this case report publication.

\section{References}

1. Backman T, Rauramo I, Huhtala S, Koskenvuo M. Pregnancy during the use of levonorgestrel intrauterine system. American Journal of Obstetrics and Gynecology. 2004 1;190(1):50-4.

2. Grimbizis GF, Camus M, Tarlatzis BC, Bontis JN, Devroey P. Clinical implications of uterine malformations and hysteroscopic treatment results. Hum Reprod Update. 2001 1;7(2):161-74.

3. Letterie G S. Management of congenital uterine abnormalities. Reproductive BioMedicine Online. 2011 23(1):40-52.

4. Homer HA, Li T-C, Cooke ID. The septate uterus: a review of management and reproductive outcome. Fertility and Sterility. 2000 1;73(1):1-14.

5. Grimbizis G, Camus M, Clasen K, Tournaye H, De Munck L, Devroey P. Hysteroscopic septum resection in patients with recurrent abortions or infertility. Hum Reprod. 1998 1;13(5):1188-93.

6. Roy KK, Singla S, Baruah J, Kumar S, Sharma JB, Karmakar D. Reproductive outcome following hysteroscopic septal resection in patients with infertility and recurrent abortions. Arch Gynecol Obstet. 2011 1;283(2):273-9.

7. Selvaraj P, Selvaraj K. Reproductive outcome of septate uterus following hysteroscopic septum resection. J Hum Reprod Sci. 2010;3(3):143-5.

8. Fedele L, Bianchi S, Marchini M, Franchi D, Tozzi L, Dorta M. Ultrastructural aspects of endometrium in infertile women with septate uterus** Presented at the 50th Annual Meeting of The American Fertility Society, San Antonio, Texas, November 5 to 10, 1994. Fertility and Sterility. 1996 1;65(4):750-2.

9. Hickok LR. Hysteroscopic treatment of the uterine septum: A clinician's experience. American Journal of Obstetrics \& Gynecology. 2000 
1;182(6):1414-20.

10. March CM. Asherman's Syndrome. Semin Reprod Med. 2011 29(2):83-94.

11. Tonguc EA, Var T, Yilmaz N, Batioglu S. Intrauterine device or estrogen treatment after hysteroscopic uterine septum resection. International Journal of Gynecology \& Obstetrics. 2010;109(3):226-9.

12. Di Spiezio Sardo A, Calagna G, Scognamiglio M, O’Donovan P, Campo R, De Wilde RL. Prevention of intrauterine post-surgical adhesions in hysteroscopy. A systematic review. European Journal of Obstetrics \& Gynecology and Reproductive Biology. 2016 1;203:182-92.

13. Xue J, Zhang H, Cui M, Li N, Liu M, Mu Y. Comparative analysis of intrauterine device placement or estrogen therapy to patients after hysteroscopic uterine septum resection. Biomedical Research [Internet]. 2018 [cited 2019 0ct 15];29(10). Available from: https://www.alliedacademies.org/abstract/ comparative-analysis-of-intrauterine-device-placement-or-estrogen-therapy-to-patients-after-hys- teroscopic-uterine-septum-resection-10293.html

14. Bosteels J, Weyers S, D'Hooghe TM, Torrance H, Broekmans FJ, Chua SJ, et al. Anti-adhesion therapy following operative hysteroscopy for treatment of female subfertility (Review). Cochrane Library. 2017;(11, Art.No. CD011110):1-23.

15. The National Institute for Health and Care Excellence (NICE). Overview | Hysteroscopic metroplasty of a uterine septum for primary infertility [Internet]. 2015 [cited 2019 Nov 3]. Available from: https://www.nice.org.uk/guidance/ipg509

16. Hopkins MR, Agudelo-Suarez P, El-Nashar SA, Creedon DJ, Rose CH, Famuyide AO. Term pregnancy with intraperitoneal levonorgestrel intrauterine system: a case report and review of the literature. Contraception. 2009 1;79(4):323-7.

Received 04-12-20

Revised 10-12-20

Accepted 15-12-20 